\title{
Novel Integration of Wi-Fi Signal and Magnetometer Sensor Measurements in Fingerprinting Technique for Indoors Smartphone positioning
}

\author{
Runahi Friad Qadr ${ }^{1}$, Halgurd S. Maghdid ${ }^{2 *}$, and Azhin T. Sabir ${ }^{2}$ \\ ${ }^{1}$ MSc Student at Department of Software Engineering, Faculty of Engineering, Koya University, \\ Kurdistan Region-F.R.Iraq. email: runahi.software@gmail.com \\ 2 Department of Software Engineering, Faculty of Engineering, Koya University, Kurdistan Region- \\ F.R.Iraq. emails: first.last@koyauniversity.org.
}

\begin{abstract}
Smartphones are becoming more widespread, and locationbased services (LBS) have become one of the most important uses in people's daily lives. While outdoor location is reasonably simple thanks to GNSS signals, however, indoor location is more problematic due to the lack of GNSS signals. As a result of the widespread deployment of alternative technologies such as wireless and sensors technologies, various studies on wireless-based indoor positioning have been conducted. However, each technology has its own limitations including multipath fading of wireless signals causes time-varying received signal strength as well as the accumulated error of the onboard sensors (i.e. sensor drift) resulting in poor localization accuracy. Motivated by these restrictions, this work integrates the applicability of two technologies for indoor positioning that are already available in smartphones by avoiding their limitation. The integration is based on fingerprinting-positioning technique by including magnetometer sensor measurements and WiFi signal strength. Androidbased smartphones with low-cost sensors in real indoor scenarios are utilized to create a dataset and collect independent track tests to confirm results. The performance of different scenarios, such as Wi-Fi alone, magnetometer alone, and magnetometer-aided Wi-Fi, is compared. The experimental results show that the combination of magnetometer sensor and WiFi signal strength provides significant results in which leads to reducing the location error to 0.7224 meters.
\end{abstract}

\section{Introduction}

Utilizing smartphone applications to run daily life activities via users is increased day-byday. For example, billing of foods/water/electricity, navigation in popular or unknown venues, tracking patient in hospitals, travel through airports, news and commercial advertisement, and sport/fitness activities are the most well-known applications on today's

* Corresponding author: halgurd.maghdid@koyauniversity.org 
smartphones [1]. The unique feature of these applications is using location information. Further, indoor location information has acquired a huge attention as the demand for Location-Based Services (LBS) users via smartphones [2]. There has been a huge of number of techniques and technologies to run LBS applications. Pedestrian dead-reckoning with inertial sensors, fingerprinting with $\mathrm{Wi}-\mathrm{Fi}$, trilateration with beacon signals, and integration with high-sensitivity Global Navigation Satellite System (GNSS) receivers are only a few examples of prevalent techniques/technologies. Specifically, two types of positioning techniques based on Received Signal Strength (RSS) via WiFi technology can be implemented: 1) trilateration with a route loss model and 2) fingerprinting with a dataset. The first relies significantly on the signal propagation model and requires WiFi access point (WAP) locations. Fingerprinting, on the other hand, makes no such assumptions and uses a training dataset to calculate the likelihood of a user's location. In order to determine a user's location based on fingerprinting, various metrics can also be utilized to pattern-match between the online measurements and stored measurements in the dataset [3]. There are two phases in traditional fingerprinting which are offline and online phases [4]. The data collection method is carried out in an environment during the offline phase. The coordinates of RPs, as well as their respective signal intensities from Wi-Fi access points, are gathered. During the online phase, the indoor positioning algorithm estimates a location based on currently observed signal strengths and previously gathered information from RPs [5]. The received signal intensity can be modified by diffraction, reflection, scattering, and absorption during propagation in indoor situations, which is the main problem for positioning algorithms based on location fingerprinting, also because of the magnetic field's stability and distinctiveness, a number magnetic-based positioning techniques have been developed [6]. The abundance of WAPs in some interior areas, such as office buildings and shopping malls, allows the fingerprinting technology to function well [7]. As a result, Wi-Fi RSS-based fingerprinting location has grown popular, however the RSS measurements are not stable due to: 1) WiFi signal interference, WiFi chipset reading errors (i.e. $\pm 4 \mathrm{dBm}$ ), and WiFi signal coverage in the vicinity [8]. Furthermore, with inertial sensors such as magnetometer sensor, the inside magnetic field anomalies induced by structural steel elements impact orientation estimate, although it is a signature for localization purposes in the meantime [9]. It is clear from the literature review that the majority of indoor positioning tests necessitate additional equipment installation [10-12] or they provide low positioning accuracy $[13,14]$. To the best of our knowledge, so far, there is no a perfect solution for indoor localization while this work proposed an algorithm that combines features and advantages of RSS and magnetometer sensor together to accomplish or to provide better positioning accuracy at lower cost of survey of the area

Therefore, the key contribution of this work is: an integration of the benefits of combining magnetometer sensor measurements and Wi-Fi RSS values for positioning.

The rest sections of the paper are organized as the following, section 2 states the problem of fingerprinting positioning technique as well as investigate the current related solution. The proposed algorithm is deeply described in Section 3. Section 4 shows various trail experiments to demonstrate the validity of the proposed approach. Finally, section 5 summarizes the major accomplishments and outlines future plans.

\section{The Proposed Algorithm}

This study proposed a new integration of WiFi RSS values with magnetometer sensor measurements into fingerprint positioning techniques. The proposed approach this work is shown in Figure 1. The proposal via fingerprint technique for indoor positioning consists of two main phases which are offline and online phases. In the offline phase, the RSS values of WAPs and three axis magnetometer sensor measurements are collected in a site 
survey along the planned path. Further, the RSS values of WiFi access points and the magnetometer sensor measurements are used as positioning features which are gathered and stored with their relation location information ( $\mathrm{x}$ and $\mathrm{y}$ coordinated) into the fingerprint dataset. An example of the gathered features during the survey is expressed in equation (1).

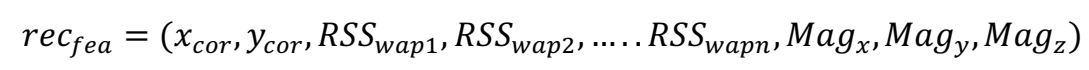

Where $\mathrm{x}_{\text {cor }}$ and $\mathrm{y}_{\text {cor }}$ are the location information at the fingerprint survey, $\mathrm{RSS}_{\mathrm{wap} 1}, \mathrm{RSS}_{\mathrm{wap} 2}, \ldots . . \mathrm{RSS}_{\mathrm{wapn}}$ are the RSS values of the WAPs signals in the vicinity, and finally the $\operatorname{Mag}_{\mathrm{x}}, \mathrm{Mag}_{\mathrm{y}}, \mathrm{Mag}_{\mathrm{z}}$ are the three axis magnetometer measurements.

In online phase, the new RSS values of the WAPs and magnetometer measurements are then compared to the dataset's stored recorded values using the k-NN algorithm. Within k$\mathrm{NN}$, the comparison is applied by calculating the distance between the new RSS and magnetometer measurements and all previously stored or recorded data. The distance is determined in based on using the Euclidean distance, as shown in equation (2). Because of Android OS is free, powerful, and full, Android-based smartphone is utilized to collect the data records by enabling WiFi chipset and onboard magnetometer sensor

$$
d_{i}=\sqrt{\left(O N_{-} R S S_{-} M a g-O F_{-} R S S_{-} M a g_{i}\right)^{2}}
$$

Where $d_{i j}$ is the calculated distance between the online and offline values for the ith record, OF_RSSi_Mag ${ }_{i}$ is the offline RSS and magnetic values at ith record, and ON_RSS_Mag is the online record values.

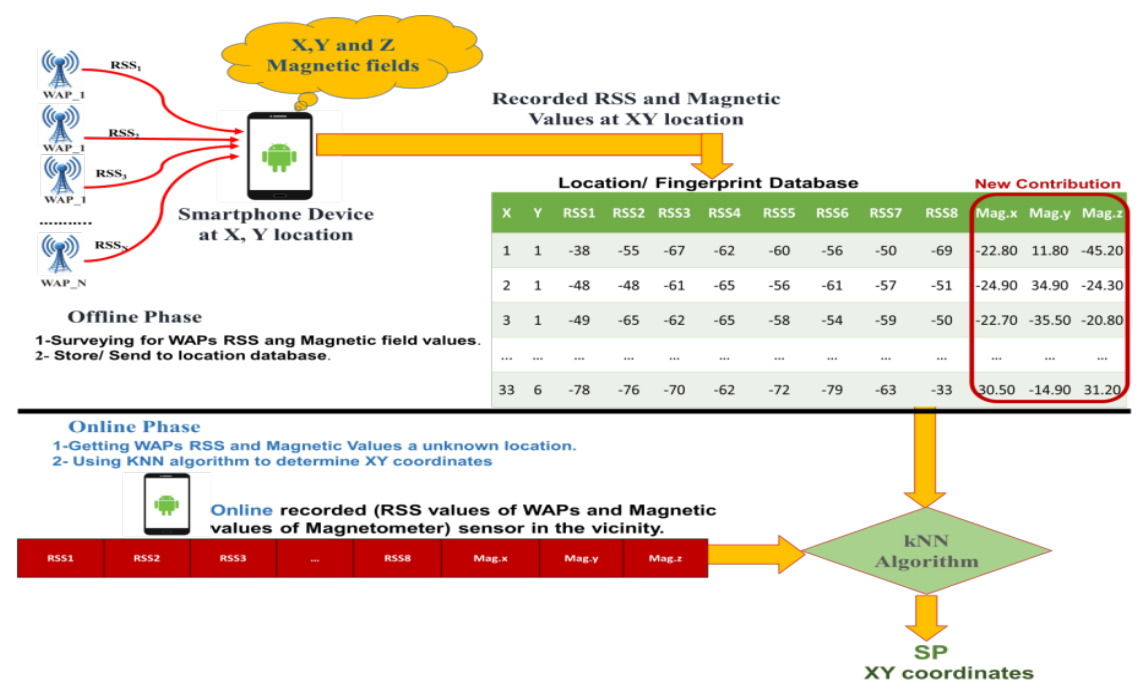

Fig. 1. The diagram of the proposed approach.

The distances for all the stored records are calculated, then in ascending order, the distances are sorted. Because of the real-time RSS values would be closed to some of the pre-stored RSS records, the first $\mathrm{k}$ distances are chosen. The $\mathrm{k}$-value is determined via a fingerprint survey of the surrounding area or sites. Finally, for calculating smartphone (x, y) coordinates of the corresponding coordinates of the k-selected distances are used as the estimated smartphone location. 


\section{Dataset and Experimental Setup}

To show the performance of the proposed approach, this study is tried to create a new dataset. The dataset is constructed via a survey at the first floor of the Koya Private Institute for Computer Science (Koya-PICS) building. The survey area is around $33 \mathrm{~m}$ by $6 \mathrm{~m}$. At each meter square (at each fingerprint) the Wi-Fi RSS values and magnetometer measurements are collected. Thus, the number of the recorded data is around 198 samples. Further, due to instability of the WiFi RSS values and magnetometer values, this study attempts to get the values by three times at each fingerprint. Therefore, the total recorded data, in the offline phase, is equal to 594 records. Note the record sample is arranged as expressed in equation (1). During the survey, the RSS values of WAPs and magnetometer measurements are collected using an Android application on a smartphone.

To evaluate the proposed approach, MATLAB packages is used. A set of experimented is carried out. First, to provide truth fingerprint which has been used during the positioning accuracy, a MATLAB-simulated graph based on real survey of the building is shown in figure 2. The WAPs RSS in the vicinity, magnetometer measurements, and $\mathrm{x}$, y coordinate values of the fingerprints are then stored. Next, as an initial experiment, the WAPs RSS values are depicted in figure 3. This is to show how the values of WAPs RSS values are changed along the path of the survey. As shown, there are eight WAPs RSS values.

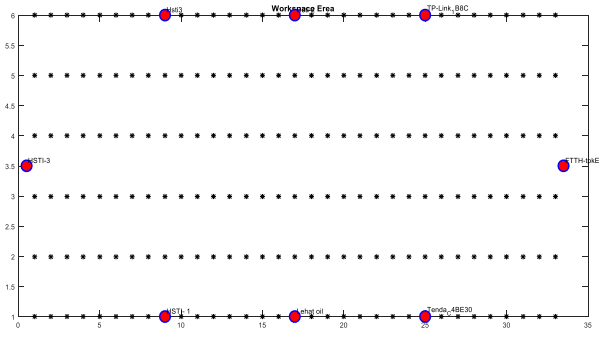

Fig. 3. A simulated-MATLAB graph of the real survey for the Koya-PICS building.

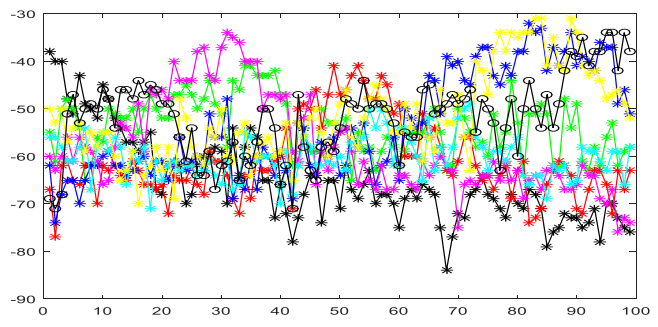

Fig. 2. The eight WAPs RSS values along the survey path.

Moreover, to understand how the proposed integration approach is working, three different experimental scenarios are conducted. The first scenario is to conduct an experiment to use the WAPs RSS values of the dataset. The second scenario is to test an experiment to use only the collected magnetometer measurements of the dataset. The last scenario is to integrate both WAPs RSS values and magnetometer measurements of the dataset. Note, the proposed approach is also implemented when the measured WAPs RSS values and magnetometer measurements values with fingerprint information are available to demonstrate the validity of the algorithm performance. The process of matching between offline WAPs RSS values and online WAPs RSS values is handled by the algorithm using the k-NN technique. As described in previous section, the position of the smartphone is estimated using equation 3 .

$$
\mathrm{X}_{\mathrm{es}}=\frac{\sum_{\mathrm{i}}^{\mathrm{k} X i}}{\mathrm{~K}}, \mathrm{Y}_{\mathrm{es}}=\frac{\sum_{\mathrm{i}}^{\mathrm{k}} \mathrm{Yi}}{\mathrm{K}}
$$

Where $\mathrm{k}$ is the number of selected records and $\mathrm{x}$ and $\mathrm{y}$ is the coordinates of the smartphone position in the pre-surveyed map and Yes and Xes is the estimated position. Note, the positioning accuracy via the root mean square error (RMSE) is used to evaluate the performance of the experimental scenarios, as indicated in equation (4).

$$
\operatorname{pos}_{\mathrm{err}}=\sqrt{\frac{1}{2}\left(\mathrm{X}_{\mathrm{es}}-\mathrm{X}_{\mathrm{t}}\right)^{2}+\left(\mathrm{Y}_{\mathrm{es}}-\mathrm{Y}_{\mathrm{t}}\right)^{2}}
$$

Where $\operatorname{pos}_{\mathrm{err}}$ is the positioning accuracy, $\mathrm{X}_{\mathrm{es}}$ and $\mathrm{Y}_{\mathrm{es}}$ are the estimated $\mathrm{X}$ and $\mathrm{Y}$ location coordinates, and $\mathrm{X}_{\mathrm{t}}$ and $\mathrm{Y}_{\mathrm{t}}$ are truth values of $\mathrm{X}$ and $\mathrm{Y}$ location coordinates respectively. 


\section{Results and Discussions}

For the sake of the performance of the proposed integration, each technology (including $\mathrm{WiFi}$ and magnetometer) has its own strengths and weaknesses based on its unique properties. For example, WAPs signal readings and magnetometer measurements can provide location information based on different positioning techniques. However, the primary challenge with using magnetometer sensor measurements in indoor environments is the issue of interference caused by building structures or steel obstructions. Also, WAPs signals will be affected by exiting complex indoors structure such as: thickness of walls, doors, and ceiling. In addition to that, the movable object including people moving in the vicinity will affected the WAPs signal. In contrast to the limitations of a single technologybased localization system, it may take the advantages of their strengths by integrating the technology measurements into the fingerprinting technique based on their qualities. First, this study is going to through how each technology measurements in the proposed positioning approach provides positioning accuracy.

From the experiments, with the first scenario, the WAPs RSS-based fingerprint technique can estimate smartphone location by using eight WAPs RSS values from eight access points. This is followed by comparing them to the RSS value of a test sample. The average positioning error is 1.6164 meters. With the second scenario, when only magnetometer measurements including ( $\mathrm{x}, \mathrm{y}$ and $\mathrm{z}$ ) are used, the average error of the positioning accuracy is up to 3.4600 meters . This means that the obtained positioning accuracy via WAPs RSS values is better than of using magnetometer measurements. The third scenario is to test the integration positioning approach by combining the two localization technologies measurements, as it is mentioned earlier. The integration is based on taking the advantages of both $\mathrm{WiFi}$ technologies and magnetometer sensors measurements. The average positioning error which has been obtained from combining these two localization technologies is 0.7224 meter that's quite less than using each of them alone-based technology. The cumulative distribute functions (CDF) of the achieved positioning accuracy (positioning error) and the positioning error at each fingerprint of the dataset are shown in figure 4 and figure 5 , respectively.

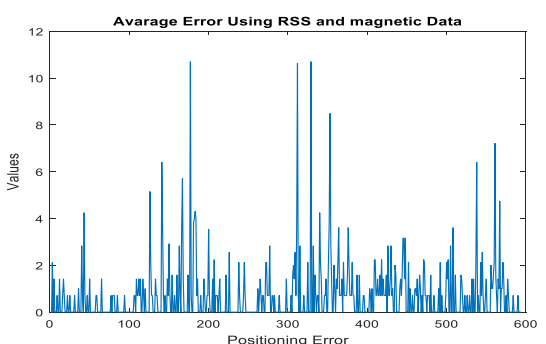

Fig. 5. Positioning error for all the fingerprints of the dataset when both WAPs RSS values and magnetometer is used.

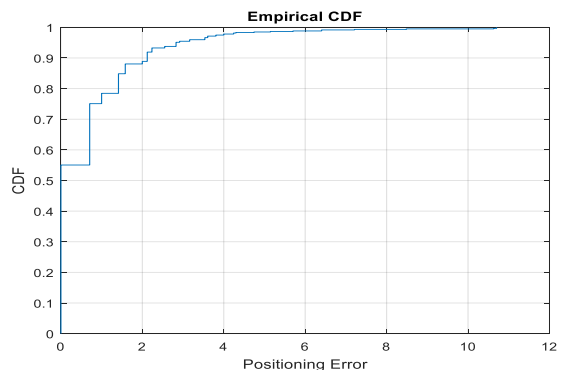

Fig. 4. Cumulative distributions function of the positioning error when both Magnetometer and RSS is used] measurements is used

\section{Conclusion}

In this study an integration indoor positioning technique using Wi-Fi signals and magnetic field of magnetometer sensor is presented. The integration approach is to improve smartphone position using error minimization and to provide better positioning accuracy. The integration is also compared to fingerprinting technique using WiFi technology and magnetometer measurements separately, as they have possibility of realization in practice. Further, magnetic based navigation system does not need any infrastructure and just 
requires the magnetic fingerprint map. With this work a set of experiments and different scenarios are conducted to prove the validity of the proposed approach. The experimental results showed that the average positioning errors provided by WiFi RSS values and magnetometer sensors are 1.6662 and 3.74 meters, respectively. Further to effectively localize the position and to improve the positioning accuracy, this study proposed the integration of these two technologies. The experimental results of the integration, shows that better performance can be achieved by, minimizing the smartphone positioning error to 0.7224 meter. Future steps of this study could be based on modifying the k-NN algorithm (as it has been used as a matching algorithm in the fingerprinting technique). The modification is to use feature weighting method to combine RSS values and magnetometer measurements. In addition, some statistical procedures can be applied on the WAPs RSS values and magnetometer measurements to improve the matching process via k-NN or any machine learning algorithms including decision tree, SVM, or deep learning algorithms.

\section{References}

1. Ali, M.U., S. Hur, and Y.J.S. Park, Wi-Fi-based effortless indoor positioning system using IoT sensors. 2019. 19(7): p. 1496.

2. Ninh, D.B., et al., An effective random statistical method for Indoor Positioning System using WiFi fingerprinting. 2020. 109: p. 238-248.

3. Mendoza-Silva, G.M., et al., Long-term WiFi fingerprinting dataset for research on robust indoor positioning. 2018. 3(1): p. 3.

4. Ashraf, I., S. Hur, and Y.J.S. Park, Indoor positioning on disparate commercial smartphones using Wi-Fi access points coverage area. 2019. 19(19): p. 4351.

5. Ali, W.H., A.A. Kareem, and M.J.C.U.-E.S.J. Jasim, Survey on wireless indoor positioning systems. 2019. 3(2): p. 42-47.

6. Yeh, S.-C., et al., Study on an indoor positioning system using Earth's magnetic field. 2019. 69(3): p. 865-872.

7. Hanley, D., et al., The Impact of Height on Indoor Positioning With Magnetic Fields. 2021. 70: p. 1-19.

8. Hosseini, K.S., et al. Improving Multi-floor WiFi-based Indoor positioning systems by Fingerprint grouping. in 2021 th International Conference on Internet of Things and Applications (IoT). 2021. IEEE.

9. Kuang, J., T. Li, and X.J.I.S.J. Niu, Magnetometer bias insensitive magnetic field matching based on pedestrian dead reckoning for smartphone indoor positioning. 2021.

10. Dari, Y.E., S. Suyoto, and P.J.I.J.I.M.T. Pranowo, CAPTURE: A Mobile Based Indoor Positioning System using Wireless Indoor Positioning System. 2018. 12(1): p. 61-72.

11. Keser, S.B., A. Yazici, and S.J.M.I.S. Günal, An F-Score-Weighted Indoor Positioning Algorithm Integrating WiFi and Magnetic Field Fingerprints. 2018. 2018: p. 7950985:1-7950985:8.

12. Pérez-Navarro, A., et al., Challenges of fingerprinting in indoor positioning and navigation, in Geographical and Fingerprinting Data to Create Systems for Indoor Positioning and Indoor/Outdoor Navigation. 2019, Elsevier. p. 1-20.

13. Ashraf, I., et al., A Comprehensive Analysis of Magnetic Field Based Indoor Positioning With Smartphones: Opportunities, Challenges and Practical Limitations. 2020. 8: p. 228548-228571.

14. Gozick, B., et al., Magnetic maps for indoor navigation. 2011. 60(12): p. 3883-3891. 